\title{
Blood indices, in-hospital outcome and short-term prognosis in patients with COVID-19 pneumonia
}

\author{
Karrar Al-Buthabhak ${ }^{1}$, Hussein Nafakhi ${ }^{1}$, Mohammed H Shukur ${ }^{2}$, Ahmed Nafakhi ${ }^{3}$, \\ Mohammed Alareedh ${ }^{1}$, Foaad Shaghee ${ }^{4}$ \\ ${ }^{1}$ Internal Medicine Department, Medicine College, University of Kufa, Najaf; ${ }^{2}$ Al-Sader Teaching Hospital, Najaf \\ Health Bureau, Ministry of Health, Najaf; ${ }^{3}$ Research Unit, Najaf Health Bureau, Ministry of Health, Najaf; ${ }^{4}$ Internal \\ Medicine Department, Faculty of Medicine, Jabir ibn Hayyan Medical University, Kufa, Iraq
}

\begin{abstract}
The predictive role of blood indices in coronavirus disease 2019 (COVID-19) related in-hospital adverse outcomes and post-recovery status is not fully defined. The main aim was to assess the association of complete blood indices measured at baseline with COVID-19 related in-hospital clinical outcomes, including length of hospital and intensive care unit (ICU) stay, receiving mechanical ventilation, degree of lung injury and in-hospital death, and postrecovery status. This retrospective study included patients with newly diagnosed COVID-19 infection from August 20, to September 25, 2020. The initial study cohort included 127 patients
\end{abstract}

Correspondence: Hussein Nafakhi, Internal Medicine Department, Medicine College, University of Kufa, P.O. 21, Kufa, Najaf, Iraq. Tel. +964.78.2184116 - Fax: +964.340952.

E-mail: husseinaf.alnaffakh@uokufa.edu.iq

Key words: COVID-19; in-hospital death; blood indices; outcome; prognosis.

Authors' contributions: KA, MS, AN data acquisition; HN, KA, data analysis, manuscript drafting; HN, FS, KA data interpretation; HN, KA, FS, MA manuscript critical revision for important intellectual content; HN, MA, KA conception and design of the work. All the authors have read and approved the final version of the manuscript and agreed to be accountable for all aspects of the work.

Conflict of interest: The authors declare that they have no conflict of interest.

Ethics approval and informed consent: Approval of this study was provided by our medicine College Board. Data were collected and analysed only by researchers, providing full confidentiality.

Received for publication: 26 January 2021.

Accepted for publication: 30 April 2021.

COCopyright: the Author(s), 2021

Licensee PAGEPress, Italy

Monaldi Archives for Chest Disease 2021; $91: 1782$

doi: 10.4081/monaldi.2021.1782

This article is distributed under the terms of the Creative Commons Attribution Noncommercial License (by-nc 4.0) which permits any noncommercial use, distribution, and reproduction in any medium, provided the original author(s) and source are credited. with newly diagnosed COVID-19. Of whom 26 patients were excluded, leaving 101 patients for final analysis. low lymphocytes $\%$ [Odds ratio and confidence intervals $=$ OR $(\mathrm{CI})][0.2(0.0-0.2$, $\mathrm{p}=0.03]$ increased the odds of ICU stay length while high platelet mean volume (PMV) $[0.9(1.1-5, \mathrm{p}<0.00]$, high platelet distribution width (PDW) [0.3(0.4-1.9), $\mathrm{p}<0.00]$, and low lymphocytes \% [0.2 $(0.0-0.2), p=0.02]$ increased the odds of length of hospital stay. Decreased lymphocytes \% showed significant independent association with increased risk for mechanical ventilation use [0.9 (0.9-1), $\mathrm{p}=0.04]$, extensive degree of lung injury [0.2 (0.1-0.7), $\mathrm{p}<0.00]$, and in-hospital death $[0.5(0.3-0.8), \mathrm{p}=0.01]$. High lymphocytes $\%[0.9$ $(0.9-1), \mathrm{p}<0.00]$ and high PMV $[0.3(0.3-0.8), \mathrm{p}=0.02]$ were significantly associated with complete recovery while increased neutrophil \% [1 (1-1.1), p=0.04] was associated with increased risk for post recovery fatigue. In conclusion, low lymphocytes $\%$ and high neutrophil $\%$ are useful markers for predicting adverse in- hospital outcome and post-recovery persistent fatigue, respectively. High PMV and lymphocyte \% showed significant association with favorable short term prognosis.

\section{Introduction}

Severe acute respiratory syndrome coronavirus 2 (SARS-CoV2) causing coronavirus disease 2019 (COVID-19) has rapidly become a global pandemic and public health problem in almost all countries.[1] COVID-19 infection has broad clinical spectrum, encompassing asymptomatic infection, mild upper respiratory tract illness, severe viral pneumonia with respiratory failure, and even death [1-3]. The exact pathophysiological mechanism responsible for severe and critically ill cases of COVID-19 infection is still not fully understood. It has been suggested that an immunological, hematological and inflammatory changes may play a major role in the rapid progression from mild to critical COVID-19 [2].

Routine complete blood analysis is inexpensive, widely available and valuable tool which can be used for assessment of the systemic inflammatory response related COVID-19 infection and may help to discriminate between patients with or without severe disease [4]. Several studies have demonstrated the correlation of blood indices abnormalities, such as neutrophils, lymphocytes, and platelets with COVID-19 diagnosis and severity [4-6]. However, the predictive role of various blood indices in COVID-19 related inhospital adverse outcomes and post-recovery status is undefined and poorly described in the literature, which needs further investigation to expand our knowledge about COVID-19 burden.

The main aim of the present study was to assess the association 
of complete blood indices measured at baseline with COVID-19 related in-hospital clinical outcomes, including length of hospital and intensive care unit (ICU) stay, receiving mechanical ventilation, degree of lung injury and in-hospital death, and post-recovery status after 14-day from resolution of fever or discharge from the hospital.

\section{Materials and Methods}

This was an observational retrospective study included patients with newly diagnosed COVID-19 infection who presented to the outpatients' clinic or admitted to the Al-Sader teaching hospital in Al-Najaf governorate from August 20, to September 25, 2020. All patients were presented with features consistent with COVID-19 pneumonia based on clinical symptoms (fever, cough, sputum or shortness of breath) and radiological findings. Patients diagnosed with COVID-19 according to positive nasopharyngeal swab by real time polymerase chain reaction (PCR). At hospital admission, the baseline clinical characteristics and complete blood count were recorded using medical records and collected by physicians at study site-level. At outpatient clinic, the evaluation of patients was performed by physicians through the clinical interview with the patient during outpatient clinic visit. The baseline clinical characteristics including age, sex, hypertension, diabetes mellitus, chronic lung disease, smoking, body mass index (BMI), previous coronary artery disease, complete blood count, and in-hospital clinical outcome. Complete blood indices included white blood cell count (WBC), lymphocyte count and \%, neutrophil count and \%, red blood cell count $(\mathrm{RBC})$, hemoglobin $(\mathrm{Hb})$, red blood cell mean volume $(\mathrm{MCV})$, red blood cell width distribution (RDW), platelet count, platelet distribution width (PDW), and platelet mean volume (PMV). The severity of lung damage by COVID-19 pneumonia was assessed by chest computed tomography (CT) scan score (Aquilon 64, v. 4.51 ER 010; Toshiba Medical Systems, Tochigi, Japan) at the time of hospital admission or outpatient clinic. According to pneumonia severity and radiological features of lung injury, patients with mild pneumonia were treated at home and patients with moderatesevere pneumonia were admitted to the hospital and followed up until discharge or death. All patients, whether admitted to hospital or treated at home, must have a second visit to outpatient clinic after 14 days from resolution of fever related to COVID-19 pneumonia or discharge from the hospital to assess recovery status and post-recovery persistent or new symptoms. Patients with incomplete data or discharge on their responsibility before completion of treatment or not attended 14-day post discharge or recovery visit were excluded. The main outcome for the study was defined as receiving mechanical ventilation, length of ICU stay, duration of hospital admission, degree of lung damage according to CT score, in-hospital death, complete recovery, and partial recovery with persistent symptoms. Approval of this study was provided by our Medicine College Board.

\section{Statistical analysis}

Statistical analysis was performed using SPSS (Statistical Package for the Social Sciences) ver. 23.0 (SPSS Inc., Chicago, IL, USA). p-value of $<0.05$ was chosen for statistical significance. Baseline clinical data of the patients and blood indices were expressed as mean \pm standard deviation for continuous variables or as numbers with percentages for categorical data. Univariate and multivariate logistic regression analyses were used to calculate the odds ratio and confidence intervals $[\mathrm{OR}(\mathrm{CI})]$ and assess the association of complete blood count indices and baseline characteristics with in-hospital outcomes including length of hospital and ICU stay, degree of lung damage according to CT score, receiving mechanical ventilation, and in-hospital death and post- recovery status. Baseline clinical characteristics, including age, sex, hypertension, diabetes mellitus, chronic lung disease, smoking, BMI, previous coronary artery disease, and complete blood indices underwent univariable logistic regression to the in- hospital outcomes and post-recovery status. Those with a $p$-value of $<0.05$ were candidates for inclusion in the final multivariable logistic regression analysis.

\section{Results}

The initial study cohort included 127 patients with newly diagnosed COVID-19 who visited the outpatient clinic or admitted to the hospital with features consistent with pneumonia. Of whom 26 patients were excluded because of nonattendance for second outpatient clinic visit after 14-day from resolution of fever or hospital discharge $(n=20)$, discharge on their responsibility before completion of treatment $(n=4)$ or refuse receiving intubation $(n=2)$, leaving 101 patients for final analysis. Of 101 patients enrolled in the study, patients categorized into mild pneumonia $(n=64)$ who were completed their treatment and follow up at home and moderate-severe pneumonia $(n=37)$ who admitted to hospital, according to the clinical severity of pneumonia and radiological findings.no one of patients with mild pneumonia required hospital admission. Regarding 37 patients with moderate-severe pneumonia requiring hospital admission, 24 required ICU admission, 22 receiving mechanical ventilation, and 9 died. Of the total 101 patients, excluding 9 patients with in-hospital death, 54 had complete recovery without persistent symptoms related to COVID-19 infection. Twenty-three had persistent shortness of breath requiring frequent $\mathrm{O}^{2}$ administration and 19 had persistent fatigue. Other less frequent post-recovery persistent symptoms including persistent smell loss $(n=3)$ and dizziness $(n=2)$. The baseline patients' characteristics and complete blood indices values re summarized in Table 1.

\section{Univariate analysis of blood indices and in-hospital outcome and post-recovery status}

Univariate logistic regression analysis showed that low lymphocytes \% was associated with increased risk of all in-hospital clinical outcomes $(p<0.05)$ while high neutrophils $\%$ was significantly associated with increased risk for in-hospital death $(p=0.04)$. initial PDW and PMV were associated with increased risk for prolonged length of hospital stay $(\mathrm{p}<0.00$ and $\mathrm{p}=0.04$, respectively) and ICU stay $(p<0.00$ and $p=0.03$, respectively). High initial RDW was associated with significant lung injury $(\mathrm{p}=0.03)$. There was no association between other blood indices (neutrophil count, lymphocyte count, platelet count, $\mathrm{Hb}, \mathrm{RBC}$, and $\mathrm{MCV}$ ) and in-hospital outcomes (Table 2). Regarding the association of blood indices and post-recovery status, increased initial lymphocytes $\%$ and high initial MPV were significantly associated with complete recovery $(\mathrm{p}=0.02)$ while low initial $\mathrm{Hb}$ and high initial neutrophil $\%$ were associated with increased risk for post recovery persistent shortness of breath $(\mathrm{p}=0.03$ and $\mathrm{p}=0.02$, respectively) and post-recovery persistent fatigue $(\mathrm{p}=0.04)$ 
Table 1. Patients' characteristics.

\begin{tabular}{|c|c|}
\hline Variables & $\mathrm{n}(\%)$ or mean $\pm \mathrm{SD}$ \\
\hline Age (years) & $47 \pm 16$ \\
\hline Male & $53(53 \%)$ \\
\hline BMI & $28 \pm 4$ \\
\hline Obesity (BMI $\geq 30)$ & $40(40 \%)$ \\
\hline Hypertension & $47(46.5 \%)$ \\
\hline Diabetes mellitus & $32(32 \%)$ \\
\hline Coronary artery disease & $9(9 \%)$ \\
\hline Smoking & $28(28 \%)$ \\
\hline Chronic lung disease & $23(23 \%)$ \\
\hline $\begin{array}{l}\text { Blood indices } \\
\text { WBC, } \times 10^{9} / \mathrm{L} \\
\text { Lymphocytes count, } \times 10^{9} / \mathrm{L} \\
\text { Lymphocyte percentage } \\
\text { Neutrophils count, } \times 10^{9} / \mathrm{L} \\
\text { Neutrophils percentage } \\
\text { Red blood cell count, } 10^{6} / \mathrm{L} \\
\text { Hemoglobin, g/dl } \\
\text { MCV, fl } \\
\text { RDW, \% } \\
\text { Platelets count, } \times 10^{9} / \mathrm{L} \\
\text { PDW, \% } \\
\text { PMV, fl } \\
\text { Mild pneumonia } \\
\text { Moderate-severe pneumonia }\end{array}$ & $\begin{array}{c}9.3 \pm 4 \\
1.9 \pm 1.4 \\
23 \pm 15 \\
6.7 \pm 4 \\
69 \pm 18 \\
4.6 \pm 0.6 \\
12.5 \pm 2 \\
86 \pm 6 \\
44.6 \pm 4 \\
259 \pm 100 \\
12.6 \pm 2 \\
9 \pm 1 \\
64(63 \%) \\
37(37 \%)\end{array}$ \\
\hline $\begin{array}{l}\text { In-hospital outcomes } \\
\text { Length of ICU stay } \\
\text { ICU admission, } n \\
\text { Length of hospital stay } \\
\text { Hospital stay, } n \\
\text { Mechanical ventilation use } \\
\text { Death } \\
\text { Complete recovery }\end{array}$ & $\begin{array}{c}19 \pm 9 \\
24(24 \%) \\
17 \pm 10 \\
37(37 \%) \\
22(22 \%) \\
9(9 \%) \\
54(53 \%)\end{array}$ \\
\hline $\begin{array}{l}\text { Post recovery persistent symptoms } \\
\text { Shortness of breath }\left(0^{2} \text { dependent }\right) \\
\text { Fatigue } \\
\text { Smell loss } \\
\text { Dizziness }\end{array}$ & $\begin{array}{c}23(23 \%) \\
19(19 \%) \\
3(3 \%) \\
2(2 \%)\end{array}$ \\
\hline
\end{tabular}

(Table 3). There was no association between other blood indices measured at baseline (neutrophil count, lymphocyte count, platelet count, $\mathrm{RBC}, \mathrm{MCV}, \mathrm{RDW}$, and PDW) and post- recovery status or persistent symptoms (Table 3 ).

\section{Multivariate analysis}

In a multivariable logistic regression analysis that included statistically significant univariate clinical characteristics and statistically significant univariate blood indices with in-hospital outcome and post-recovery status, we found that old age [0.6(0.1-0.5), $\mathrm{p}<0.00]$ and low lymphocytes $\%$ [0.2(0.0-0.2, $\mathrm{p}=0.03]$, increased the odds of ICU stay length while old age [0.5(0.1-0.5), $\mathrm{p}<0.00]$, high PMV [0.9(1.1-5, $<<0.00]$, high PDW [0.3(0.4-1.9), p<0.00], low lymphocytes \% [0.2(0.0-0.2), $\mathrm{p}=0.02]$ and non-smoking $[0.2(0.7-8)$, $\mathrm{p}=0.02]$ increased the odds of length of hospital stay. Decreased lymphocytes $\%$ showed significant independent association with increased risk for mechanical ventilation use [0.9(0.9-1), $\mathrm{p}=0.04]$, extensive degree of lung injury [0.2(0.1-0.7), $\mathrm{p}<0.00]$ and in-hospital death $[0.5(0.3-0.8), \mathrm{p}=0.01]$ (Table 4).

Regarding the association of significant variables in univariate analysis with recovery status, high lymphocytes \% [0.9(0.9-1), $\mathrm{p}<0.00]$, high PMV [0.3(0.3-0.8), $\mathrm{p}=0.02]$ and age less than 45 year [1(0.9-1), $\mathrm{p}=0.04]$ were significantly associated with complete recovery while old age ( $>45$ year) $[1.1(1-1.1), \mathrm{p}=0.04]$ and increased neutrophil \% [1(1-1.1), $\mathrm{p}=0.04]$ were associated with increased risk for post recovery persistent shortness of breath and fatigue, respectively (Table 5).

\section{Discussion}

Some novel findings were found. First, decreased lymphocytes $\%$ at baseline showed significant independent association with adverse in-hospital outcome, including degree of lung injury, receiving mechanical ventilation and in-hospital death. However no significant independent association was observed between decreased lymphocytes $\%$ and post-recovery persistent symptoms. On the other hand, increased neutrophil \% was significantly asso-

Table 2. Univariate regression analysis of blood indices and in-hospital clinical outcomes.

\begin{tabular}{|c|c|c|c|c|c|}
\hline & $\begin{array}{l}\text { Length of } \\
\text { ICU stay } \\
\text { p-value }\end{array}$ & $\begin{array}{l}\text { Length of } \\
\text { hospital stay } \\
\text { p-value }\end{array}$ & $\begin{array}{l}\text { Degree of } \\
\text { lung injury } \\
\text { p-value }\end{array}$ & $\begin{array}{c}\text { Mechanical } \\
\text { ventilation use } \\
\text { p-value }\end{array}$ & $\begin{array}{l}\text { In-hospital } \\
\text { death } \\
\text { p-value }\end{array}$ \\
\hline WBC & 0.81 & 0.65 & 0.68 & 0.45 & 0.60 \\
\hline Lymphocytes\% & $<0.00$ & 0.01 & $<0.00$ & $<0.00$ & $<0.00$ \\
\hline Neutrophil\% & 0.67 & 0.61 & 0.62 & 0.69 & 0.04 \\
\hline Lymphocyte count & 0.98 & 0.80 & 0.85 & 0.20 & 0.54 \\
\hline Neutrophil count & 0.75 & 0.58 & 0.76 & 0.51 & 0.56 \\
\hline $\mathrm{RBC}$ & 0.39 & 0.29 & 0.57 & 0.48 & 0.20 \\
\hline $\mathrm{Hb}$ & 0.18 & 0.16 & 0.39 & 0.36 & 0.47 \\
\hline MCV & 0.79 & 0.62 & 0.34 & 0.78 & 0.48 \\
\hline RDW & 0.56 & 0.63 & 0.03 & 0.87 & 0.22 \\
\hline Platelet count & 0.49 & 0.46 & 0.89 & 0.13 & 0.39 \\
\hline PDW & $<0.00$ & $<0.00$ & 0.25 & 0.12 & 0.13 \\
\hline PMV & 0.03 & 0.04 & .91 & 0.32 & 0.43 \\
\hline
\end{tabular}

WBC, white blood cell count; RBC, red blood cell count; Hb, hemoglobin; MCV, mean cell volume; RDW, red blood cell distribution width; PDW, platelet distribution width; PMV, platelet mean volume. 
Table 3. Univariate regression analysis of blood indices recovery status.

\begin{tabular}{lccc} 
& $\begin{array}{c}\text { Complete recovery } \\
\text { P-value }\end{array}$ & $\begin{array}{c}\text { Post-recovery } \\
\text { shortnessof breath ( } \mathbf{O}_{2} \text { dependent) } \\
\text { p-value }\end{array}$ & 0.57 \\
WBCs & 0.18 & 0.81 & 0.96 \\
Lymphocytes \% & $<0.00$ & 0.02 & 0.94 \\
\hline Neutrophil \% & 0.24 & 0.68 & 0.04 \\
Lymphocyte count & 0.14 & 0.64 & 0.83 \\
\hline Neutrophil count & 0.20 & 0.70 & 0.97 \\
RBC & 0.58 & 0.03 & 0.33 \\
\hline Hb & 0.29 & 0.92 & 0.04 \\
MCV & 0.63 & 0.49 & 0.41 \\
\hline RDW & 0.69 & 0.38 & 0.30 \\
Platelet count & 0.36 & 0.07 & 0.27 \\
\hline PDW & 0.08 & 0.08 & 0.05 \\
PMV & 0.01 & 0.19 \\
\hline WBC,
\end{tabular}

WBC, white blood cell count; RBC, red blood cell count; Hb, hemoglobin; MCV, mean cell volume; RDW, red blood cell distribution width; PDW, platelet distribution width; PMV, platelet mean volume.

Table 4. Regression analysis of blood indices and in-hospital outcomes.

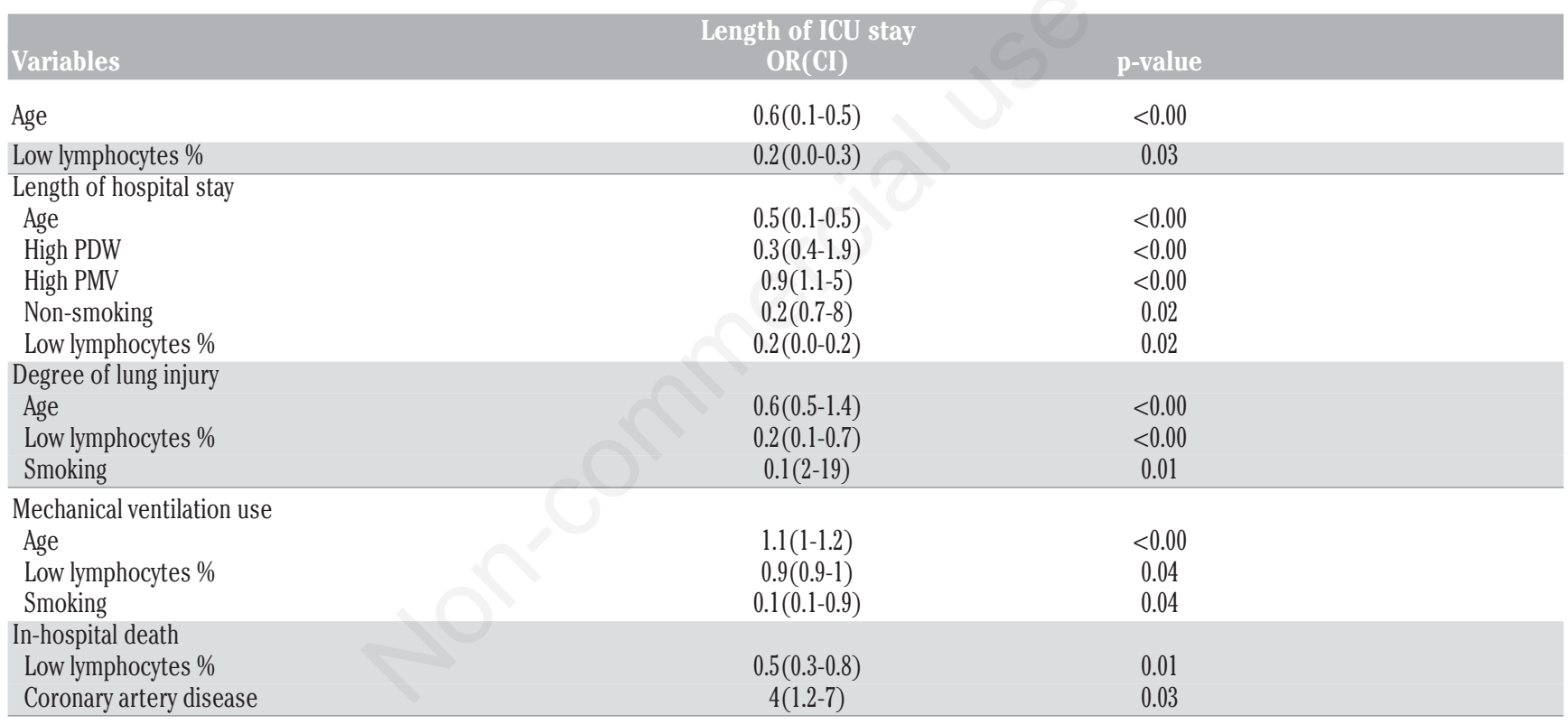

Significant variables with p-value $<0.05$ in the univariate logistic regression model were entered in the final model; Only variables significant with p-value $<0.05$ were displayed in the table. OR (CI), Odds ratio and confidence intervals; PDW, platelet distribution width; PMV, platelet mean volume.

Table 5. Regression analysis of blood indices with recovery and post recovery persistent symptoms.

\begin{tabular}{lcc} 
Complete recovery & & $<0.00$ \\
High lymphocytes \% & $0.9(0.9-1)$ & 0.02 \\
\hline High PMV & $0.3(0.3-0.8)$ & 0.04 \\
\hline Age & $1(0.9-1)$ & 0.04 \\
\hline Post recovery shortness of breath & $1.1(1-1.2)$ & 0.04 \\
\hline Age & $1(1-1.1)$ & \\
\hline $\begin{array}{l}\text { Post recovery persistent fatigue } \\
\text { High neutrophil \% }\end{array}$ & \\
\hline $\begin{array}{l}\text { Significant variables with p-value }<0.05 \text { in the univariate logistic regression model were entered in the final model; Only variables significant with p-value }<0.05 \text { were displayed in the table. OR (CI), Odds ratio and } \\
\text { confidence intervals; PMV, platelet mean volume. }\end{array}$
\end{tabular}


ciated with post-recovery persistent fatigue. Second, platelet indices of size and function, but not platelet count, showed significant independent association with prolonged length of hospital stay and complete recovery.

Because of rapid progression and considerable impact of COVID-19 infection, it is crucial to identify markers associated with predication of complications and discrimination of critical and severe cases of COVID-19 at an early stage, which are useful for early initiation of therapeutic intervention to improve shortand long-term prognosis. Notably, only a few numbers of studies reported on the outcome of COVID-19 infection, such as in-hospital adverse outcome and post-recovery persistent symptoms $[4,5,7]$

Early epidemiological studies have showed that clinical characteristics, such as age, hypertension, non-smoking, and blood indices, such as decreased lymphocyte count, increased neutrophil count, and thrombocytopenia play a role in the disease progression and severity [5,7]. A recent meta-analysis included 15 studies, which presented results of routine blood tests carried out in patients with COVID-19, found that patients with severe COVID19 had increased neutrophil \% but decreased lymphocyte and platelet counts. Further sensitivity analysis did not change the conclusion about neutrophils and lymphocytes, whereas the results regarding platelets were inconsistent [6].

The exact role of neutrophil in the pathogenesis of COVID-19 related post-recovery persistent fatigue has not been fully elucidated yet. The prolonged activation of neutrophils may lead to increased production of pro-inflammatory mediators and toxic substances, which is detrimental to the host in the short-term period [6]. Therefore, these toxic substances may be the possible explanation for the significant association of increased neutrophil $\%$ with persistent fatigue. As described previously in the literature, persistent fatigue following viral infection has been associated with a large number of differing changes in the inflammatory markers and immune cell populations $[6,8]$. Patients with persistent fatigue following viral infection have an increased rate of apoptosis, increased expression of the death receptor, and tumor necrosis factor receptor I on their neutrophils than healthy controls, which is indicative of a persistent or reactivating viral infection or a toxic state [8].

The significant association of decreased lymphocytes \% with adverse in-hospital outcomes was in line with previous studies in China, Singapore, US and Australia [9- 13]. In a meta-analysis conducted by Huang et al., the researchers found that decreased lymphocyte count was associated with threefold risk of poor outcome in severe COVID-19 infection [10]. Also, Guidelines for Australia and New Zealand, released in March 2020, reported decreased lymphocytes count and \% as prognostic marker for severe disease in COVID-19 cases [14]. Besides, decreased lymphocyte count and \% is critical factor associated with disease severity and mortality among older patients ( $>60$ years) who have more systemic symptoms [15].

A possible reason for lymphocytes reduction and destruction by SARA-COV-2 may be related to the expression of the coronavirus angiotensin-converting enzyme 2 receptor, a major link in the pathogenesis of COVID-19 leading to renin-angiotensin-aldosterone system overactivation and to increased lung damage, on the lymphocytes [6]. Another reason may be that COVID-19 infection causes the production of several inflammatory mediators and cytokines, recognizable as a cytokine storm, which leads to apoptosis of lymphocytes and atrophy of lymphoid organs $[4,16]$.

Based on the available evidence from several studies, decreased platelet count is reported to be associated with increased mortality among critically ill COVID-19 patients [3,4,17]. However, most of these studies are observational and retrospective making the correlation seen difficult to extrapolate from. Besides, a significant bulk of these studies originating in Wuhan, China, it is possible patients may have been used in more than one study as data was collected from the same location [18].

In line with our results, Manne BK et al found that non-ICU and ICU COVID-19 patients had a platelet count in the normal reference range. Also, platelet counts did not correlate with markers of inflammation. According to the results of Manne et al.'s study, platelets may be not the main contributor, as other blood cells, for increased risk of morbidity and mortality related to COVID-19 infection [19]; additionally, a prior study showed that previous strains of coronavirus had no effect on platelet counts in infected patients, suggesting COVID-19 has different effects on platelets [20].

In the present study, high PDW and PMV, but not platelet count, showed a significant association with prolonged length of hospital stay. Also, high PMV, but not PDW, was associated with increased rate of complete recovery. In the literature, increased PMV and PDW have been reported in patients with sepsis, PDW was found to be a poor prognostic marker in severe sepsis $[9,21]$. MVP, as a marker of platelet size and age, and PDW, which reflects the variance in platelet size, and is considered a marker of platelet function and activation, have shown to correlate with platelet activity and are considered a markers of platelet activity [17]. Thus, increased MPV and PDW in COVID-19 suggests that these platelets may present in hyperactive state. Consistent with our results, patients with high PDW and high MPV were significantly associated with the worse survival in a study conducted in severe and critically ill COVID-19 patients diagnosed in Huangshi City, Hubei Province, China [22]. Higher platelet turnover leads to considerable change in platelets size and increased release of young platelets, which is functionally more active than older platelets that have higher volumes leading to high MPV as is found in COVID19 patients. Thus, increased PMV may represent a sensitive indicator of the capacity of the bone marrow to compensate for platelet turnover $[9,14]$. Nevertheless, the potential underlying mechanisms linking increased MPV with good prognosis in patients with COVID-19 pneumonia in the present study, even though PMV showed a significant association with prolonged length of hospital stay during acute period of COVID-19 pneumonia, remain elusive requiring further investigation.

Our study has some limitations. Some patients were excluded from regression analysis because of incomplete data or loss of follow up second visit. Data of post-recovery persistent loss of smell $(n=3)$ and dizziness $(n=2)$ did not enter in the regression analysis due relatively very small number. The results of our study may be a potential avenue for further investigation regarding the prognostic role of platelet indices in COVID-19 progression and possible therapeutic intervention.

In conclusion, low lymphocytes $\%$ and high neutrophil $\%$ are useful markers for predicting adverse in-hospital outcome and post-recovery persistent fatigue, respectively. High PMV and lymphocyte \% showed significant association with favorable shortterm prognosis.

\section{References}

1. Zheng KI, Feng G, Liu WY, et al. Extrapulmonary complications of COVID-19: A multisystem disease? J Med Virol 2020.

2. de la Rica R, Borges M, Aranda M, et al. Low albumin levels 
are associated with poorer outcomes in a case series of COVID-19 patients in Spain: A retrospective cohort study. Microorganisms 2020;8:1106.

3. Liu Y, Sun W, Guo Y, et al. Association between platelet parameters and mortality in coronavirus disease 2019: Retrospective cohort study. Platelets 2020;31:490-6.

4. Violetis OA, Chasouraki AM, Giannou AM, Baraboutis IG. COVID-19 infection and haematological involvement: a review of epidemiology, pathophysiology and prognosis of full blood count findings. SN Compr Clin Med 2020:1-5.

5. Chen R, Sang L, Jiang M, et al. Longitudinal hematologic and immunologic variations associated with the progression of COVID-19 patients in China. J Allergy Clin Immunol 2020;146:89-100.

6. Zeng F, Li L, Zeng J, et al. Can we predict the severity of coronavirus disease 2019 with a routine blood test? Pol Arch Intern Med 2020;130:400-6.

7. Jutzeler CR, Bourguignon L, Weis CV, et al. Comorbidities, clinical signs and symptoms, laboratory findings, imaging features, treatment strategies, and outcomes in adult and pediatric patients with COVID-19: A systematic review and meta-analysis. Travel Med Infect Dis 2020;37:101825.

8. Kennedy G, Spence V, Underwood C, Belch JJ. Increased neutrophil apoptosis in chronic fatigue syndrome. J Clin Pathol 2004;57:891-3.

9. Güçlü E, Kocayiğit H, Okan HD, et al. Effect of COVID-19 on platelet count and its indices. Rev Assoc Med Bras (1992) 2020;66:1122-7.

10. Huang I, Pranata R. Lymphopenia in severe coronavirus disease-2019 (COVID- 19): systematic review and meta-analysis. J Intensive Care 2020;8:36.

11. Wang D, Hu B, Hu C, et al. Clinical characteristics of 138 hospitalized patients with 2019 novel coronavirus-infected pneumonia in Wuhan, China. JAMA 2020;323:1061.
12. Fan BE, Chong VCL, Chan SSW, et al. Hematologic parameters in patients with COVID-19 infection. Am J Hematol 2020;95:E131-4.

13. Arentz M, Yim E, Klaff L, et al. Characteristics and outcomes of 21 critically ill patients with COVID-19 in Washington State. JAMA 2020;323:1612-4.

14. Khartabil TA, Russcher H, van der Ven A, de Rijke YB. A summary of the diagnostic and prognostic value of hemocytometry markers in COVID-19 patients. Crit Rev Clin Lab Sci 2020;57:415-31.

15. Xu Z, Shi L, Wang Y, et al. Pathological findings of COVID19 associated with acute respiratory distress syndrome. Lancet Respir Med 2020;8:420-2.

16. Terpos E, Ntanasis-Stathopoulos I, Elalamy I, et al. Hematological findings and complications of COVID-19. Am J Hematol 2020;95:834-47.

17. Zhang S, Liu Y, Wang X, et al. SARS-CoV-2 binds platelet ACE2 to enhance thrombosis in COVID-19. J Hematol Oncol 2020;13:120.

18. Kermali M, Khalsa RK, Pillai K, et al. The role of biomarkers in diagnosis of COVID-19 - A systematic review. Life Sci 202 ;254:117788.

19. Manne BK, Denorme F, Middleton EA, et al. Platelet gene expression and function in patients with COVID-19. Blood 2020;136:1317-29.

20. Kim JK, Jeon JS, Kim JW, Kim GY. Correlation between abnormal platelet count and respiratory viral infection in patients from Cheonan, Korea. J Clin Lab Anal 2016;30:185-9.

21. Guclu E, Durmaz Y, Karabay O. Effect of severe sepsis on platelet count and their indices. Afr Health Sci 2013;13:333-8.

22. He J, Wei Y, Chen J, et al. Dynamic trajectory of platelet-related indicators and survival of severe COVID-19 patients. Crit Care 2020;24:607. 